\title{
ANTIMICROBIAL RESISTANCE OF BULGARIAN ISOLATES MORAXELLA CATARRHALIS DURING THE PERIOD 1999-2018
}

\author{
Raina Gergova, Rumyana Markovska. \\ Department of Medical Microbiology, Faculty of Medicine, Medical University \\ - Sofia, Bulgaria.
}

\section{SUMMARY}

The purpose of this study was to assess the levels of antimicrobial susceptibility during a period of the last 20 years among Moraxella catarrhalis isolates and to evaluate their resistance mechanisms.

Material/Methods: A total of 618 Bulgarian clinical significant isolates $M$. catarrhalis was collected during the period 1999-2018 from patients to assess the current levels of antimicrobial susceptibility and to evaluate the beta-lactam resistance mechanisms. The minimum inhibitory concentrations (MICs) by E-test were determined against: penicillin, ampicillin, amoxicillin+clavulanic acid, cefalexin, cefuroxime, cefixime, cefotaxime, levofloxacin, azithromycin, clarithromycin, tetracycline, sulfamethoxazole+trimethoprim. PCR examination of genes encoding beta-lactamases was done.

Results: Almost all (98.88\%) of the tested strains $M$. catarrhalis showed positive cefinase test and were demonstrated elevated MICs and a lack of susceptibility to penicillin, ampicillin and cefalexin. An increase of resistance to cephalosporin of second and third generations (cefixime) was found. Higher MIC values of cefuroxime and cefixime than these of cefotaxime were detected. PCRs revealed that bro- 1 genes $(94.11 \%)$ were more frequent than bro-2.

Conclusions: The results of examined 20 years period showed that nearly all Bulgarian isolates of $M$. catarrhalis in the last years become resistant to penicillins and first generation cephalosporins via beta-lactamases, encoded predominantly by bro- 1 gene. An increase of the resistance to members of second and third generation (cefixime) cephalosporin's and slower to other antimicrobials was found. The rapid development of resistance in recent years to the most popular antimicrobial agents affects the recommendations regarding the initial approach to therapy.

Keywords: Moraxella catarrhalis, beta-lactamases, bro-1, bro-2, PCR,

\section{INTRODUCTION}

Moraxella catarrhalis is an aerobic Gram-negative diplococcus that initially has been considered a harmless commensal microorganism but then has been proved high virulence of this bacterium. At now, $M$. catarrhalis is one of the three most common causes of childhood otorhinolaryngological infections and chronic cough, as well as exacerbations of chronic obstructive pulmonary disease in adult patients [1-4]. The dynamically increasing incidence of respiratory tract infections caused by this microorganism over the past 15 - 20 years is due to the rapid selection of resistance to the most used beta-lactams and the lack of vaccine yet [5-8]. The extracellular â-lactamase production has an indirect role in the pathogenesis of infection by inactivation of penicillins or cephalosporins suitable for the treatment of other co-existing with $M$. catarrhalis susceptible pathogens in polymicrobial biofilms [9, 10]. The antibiotic resistance and the biofilm formation by $M$. catarrhalis are a major reason for the ability of bacterial cells to survive and persist in vivo in the otorhinolaryngological niche or their spreading in down respiratory tract of patients with chronic obstructive disease (COPD) [11].

The aim of this study was to assess the levels of antimicrobial susceptibility during a period of the last 20 years and to evaluate the resistance mechanisms in $M$. catarrhalis to the problematic antimicrobial agents.

\section{MATERIAL AND METHODS}

\section{Bacterial strains and samples}

A collection of 618 non-duplicate strains $M$. catarrhalis isolated from Bulgarian patients with signs of respiratory tract infection, living predominantly in Sofia, aged 1-79 years during the period of January 1999 to December 2018 were collected. The clinical samples were: mucosal samples from upper respiratory tract - nasal and pharyngeal swabs, predominantly from children with otorhinological infections or/and chronic cough $(\mathrm{N}=439)$; and sputum and bronchial-alveolar lavages $(\mathrm{N}=179)$ from adult patients predominantly with COPD. Bacterial cultures were grown routinely for $24-48 \mathrm{~h}$ at $37^{\circ} \mathrm{C}$ on Columbia agar supplemented with 5\% sheep blood (Becton Dickinson, Kelberg, Germany) in an atmosphere comprising 95\% air and $5 \% \mathrm{CO}_{2}$. Pure bacterial cultures were isolated from suspected colonies with typical cultural phenotype: light gray, not hemolytic, and which can be pushed along the surface of the agar (positive "hockey disk test"). The isolates were identified as $M$. catarrhalis by microscopic morphology - 
Gram negative diplococci and positive tests for oxidase, catalase and Indoxylacetate esterase [12]. The suspected pure bacterial cultures additionally identified when need using Crystal NH (Becton Dickinson, Kelberg, Germany). Referent strains of $M$. catarrhalis were used: ATCC 25238; $\mathrm{BCCM} / \mathrm{LMG}$ 11177, $\beta$-lactamase producing, and BCCM/ LMG 11178 - $\beta$-lactamase non-producing; CIP 103772 , with brol genes and CIP 103773 with bro2.

\section{Antimicrobial susceptibility testing}

Cefinase disks (Becton Dickinson, Kelberg, Germany) were used to investigate $\beta$-lactamase production of the examined $M$. catarrhalis strains. Antimicrobial susceptibility testing was conducted using an E-test to estimate the minimum inhibitory concentrations (MICs) value (strips with antibiotics were obtained from Laboratories Pvt. Limited, Mumbai, India) in compliance with the European Committee on Antimicrobial Susceptibility Testing (EUCAST) guidelines [13]. An 0.5 McFarland suspension from pure culture İ. catarrhalis was used to inoculate an Mueller-Hinton agar plate supplemented with 5\% defibrinated horse blood, and Nicotinamide Adenin Dinucleotide 20 mg/L (BIolab Inc., Budapest, Hungary) and E-test strips were placed, and then, the sample was incubated for $18 \pm 2$ $\mathrm{h}$ at $35^{\circ} \mathrm{C}$. The breakpoints of MICs were interpreted in accordance with the EUCAST 2019. Antimicrobial susceptibility to twelve antimicrobials (penicillin, ampicillin, amoxicillin+clavulanic acid, cefalexin, cefuroxime, cefixime, cefotaxime, levofloxacin, azithromycin, clarithromycin, tetracycline, sulfamethoxazole+trimethoprim) was evaluated (Fig. 1).

Fig. 1. MICs of penicillin and azythromycin determining by E-test

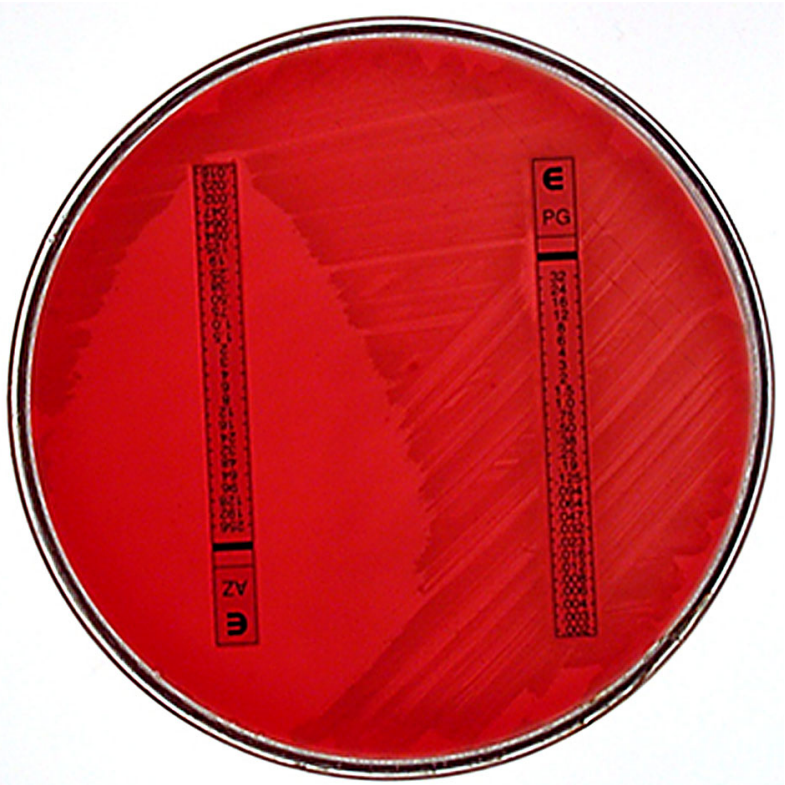

Polymerase chain reaction

For the definitive identification of the examined strains, polymerase chain reaction (PCR) with specific primers ( $c o p B$ forward GGCGTGCGTGTTGACCGTTTTG; $c o p B$ reverse GTTTGGCAGGCGATAGGCGACAT) for this bacte- rial species was performed as previously described by other authors and the amplicons with 564 bp were detected [14].

The genomic DNA was extracted using a DNA sorbAM nucleic acid extraction kit (AmpliSens, Inter Lab Service, Moscow, Russia) in accordance with the manufacturer's instructions. The pairs of primers for bro- 1 and bro-2 (5'- TTTGGATTGGGTGAATGAA -3' bro-1,2 sequences; 5'TGGGGCTGGGTGATAAATAG -3' bro-1,2 sequences) were amplified as previously described [4]. The demanded products were the following: brol (235 bp) and bro2 (214 bp), presented on Fig. 2.

Fig. 2. PCR products of bro- 1 and bro-2

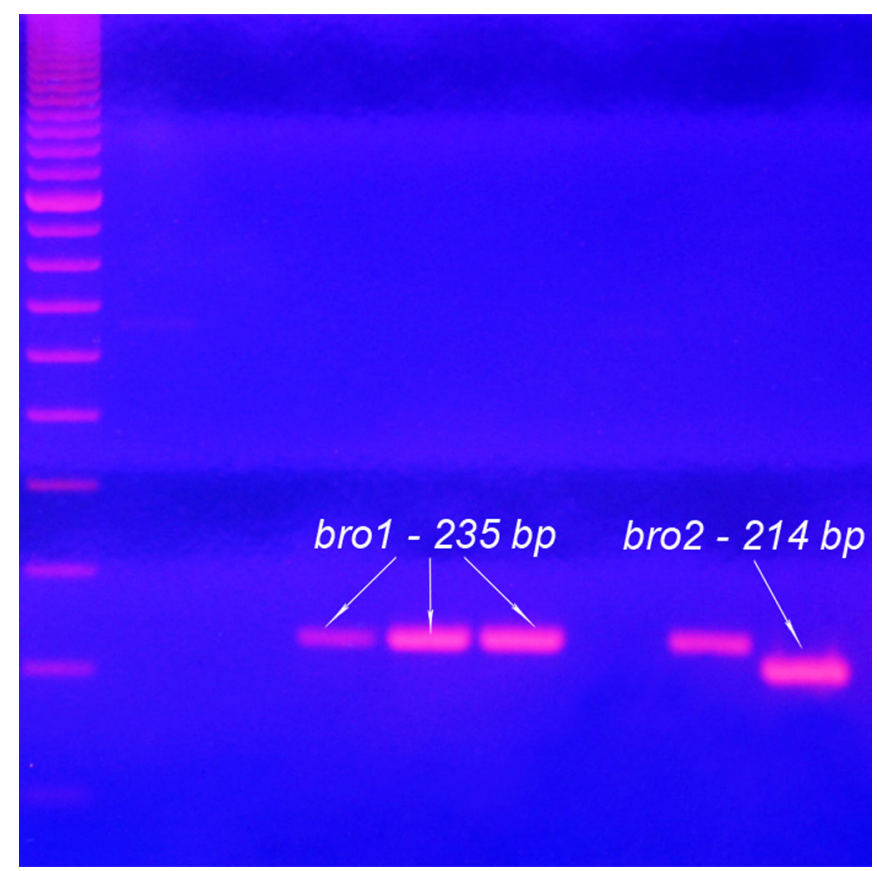

\section{STATISTICAL ANALYSIS}

Differences were analyzed using unpaired descriptive statistics, Fisher exact test Quick cal. The difference of the susceptibility rates was considered statistically significant at $p<0.05$.

\section{RESULTS}

Ninety eight point nine percent of the isolates recovered during 1999-2008, and almost $100 \%$ of those isolated between 2009-2018 were cefinase positive. The determined MICs, MIC90 of 12 antimicrobial agents and their interpretation were showed in Table1. The tested isolates M. catarrhalis were demonstrated elevated MICs and a lack of susceptibility to penicillin (Fig. 1), ampicillin and cefalexin. The distribution of antimicrobial susceptibility rates according to the years was shown in Table 2. A decrease of susceptibility rates to cefuroxime and cefixime was found. In the recent period, 2004-2018 has been detected resistance to cefotaxime at low level (Table 2). The detected susceptibility to other antimicrobials such as tetracyclines and sulfamethoxazole+trimethoprim slightly decreased during the period - from $93.4 \%$ to $88 \%$ (for tetracycline) and from $90.6 \%$ to $86.2 \%$ (for sulfamethoxazole/ trimethoprim). The PCR examination of the genetic ele- 
ments encoding beta-lactamases was showed that bro- 1 genes are more frequently detected than bro-2 $(94.11 \%$ versus $5.89 \%$ ), The derived rate of bro-l (Fig. 2) for the period $1999-2008$ was $90,83 \%$ and has become to $98.20 \%$ during 2009-2018. Some isolates were evaluated as with decreased susceptibility to macrolides, especially to clarithromycin (2,44\%). Resistant isolates $M$. catarrhalis to quinolones were not detected during the period.

Table 1. MICs of 12 antimicrobial agents to 618 strains Moraxella ñatarrhalis

\begin{tabular}{lcccc}
\hline \multirow{2}{*}{ Antimicrobial agent } & MICs & \multicolumn{3}{c}{ Interpretation } \\
& Range & $90 \%$ & $\mathrm{~S}(\%)$ & $\mathrm{R}(\%)$ \\
\hline penicillin G & $0,002->32$ & 32 & 0,97 & 99,03 \\
ampicillin & $<0,016-24$ & 8 & 0,97 & 99,03 \\
amoxicillin/Clav. acid & $<0,016-2$ & 0,25 & 100 & 0 \\
cephalexin & $0,016-32$ & 16 & 0,97 & 99,03 \\
cefuroxime & $<0,016-8$ & 2 & 63,92 & 36,08 \\
cefixime & $0,016-8$ & 4 & 69,58 & 30,42 \\
cefotaxime & $<0,016-2$ & 0,25 & 95,95 & 4,04 \\
levofloxacin & $<0,016-0,5$ & 0,06 & 100 & 0 \\
azithromycin & $<0,002-1$ & 0,06 & 99,35 & 0,65 \\
clarithromycin & $0,002-4$ & 0.25 & 97,56 & 2,44 \\
tetracycline & $0,125-48$ & 0,5 & 87,38 & 12,62 \\
trimethoprime/Sulfamethoxazole & $0,005-8$ & 0,5 & 85,76 & 14,24 \\
\hline
\end{tabular}

Table 2. Susceptibility of $618 M$. catarrhalis to antimicrobials presented in $\%$.

\begin{tabular}{lcccc}
\hline Antimicrobial agent & $\mathbf{1 9 9 9 - 2 0 0 3}$ & $\mathbf{2 0 0 4 - 2 0 0 8}$ & $\mathbf{2 0 0 9 - 2 0 1 3}$ & $\mathbf{2 0 1 4 - 2 0 1 8}$ \\
\hline penicillin & 2,9 & 1 & 1,3 & 0 \\
ampicillin & 2,9 & 1 & 1,3 & 0 \\
amoxicillin/clav.acid & 100 & 100 & 100 & 100 \\
cefalexin & 4,9 & 1,3 & 1,6 & 0 \\
cefuroxime & 97.2 & 86.7 & 79.1 & 64.9 \\
cefixime & 100 & 98.9 & 80.7 & 62.7 \\
cefotaxime & 100 & 99.4 & 98.9 & 97.1 \\
levofloxacin & 100 & 100 & 100 & 100 \\
azithromycin & 100 & 100 & 100 & 99.7 \\
clarythromyin & 100 & 100 & 100 & 98.06 \\
tetracycline & 93.4 & 93.9 & 90.6 & 88 \\
sulfomet./trimethoprime & 90.6 & 91.6 & 88.2 & 86.2 \\
\hline
\end{tabular}

\section{DISCUSSION}

The Bulgarian isolates $M$. catarrhalis have demonstrated the increase of the resistance during the last 20 years toward many beta-lactam antimicrobials due to the production of beta-lactamases, encoded predominantly by bro-1 gene. The detected MICs in the present study to penicillins and first generation cephalosporins were very high, which is in concordance with positive cefinase test for more than $98 \%$ of the isolates. This is proof of the leading role of beta-lactamases. The enzymes of $M$. catarrhalis can be divided into two closely related types, BRO1 and BRO-2. BRO-1 producing isolates have been re- ported as more resistant to beta-lactams than BRO-2 strains $[8,15]$. The higher resistance rates to penicilins and first generation cephalosporines could be explained with the high percent of bro- 1 producers (>94\%). Japan authors had found similar to our rate of bro-1 $-95 \%$, encoding the more active enzyme type and bro-2 - 5\%, respectively [8]. The increased use of beta-lactams has led to a concurrent increase in the number of pathogens with acquired resistance to this drug group. As the link between an antibiotic treatment of the host and microorganisms requires the coordinated activity of many microbial genes, this process leads to new mutations and new changes in 
the susceptibility to antimicrobials $[15,16]$. Furthermore, at last, fifteen years in Bulgaria, a decrease of susceptibility to some of the second and third generation cephalosporins (cefixime) has been detected. This could be explained most probably with the extension of the spectrum of enzymes, encoded by bla $a_{\text {bro-1. }}$. This phenomenon has been reported from other authors [8, 15-17]. More than $1 / 3$ of tested isolates $M$. catarrhalis during the last five years became resistant to the both of cefuroxime and cefixime. Similar to our results were reported by Saito et., that higher rates of MICs to cefixime and clarithromycin always associated with bro- 1 [16]. The possible extension in the present study has not affected cefotaxime - only about $4 \%$ of the isolates were with elevated cefotaxime MICs $(2 \mathrm{mg} / \mathrm{mL}) . M$. catarrhalis isolates with elevated ceftriaxone MICs $(8 \mathrm{mg} / \mathrm{mL})$ was detected via another Japanese surveillance report [17]. The isolates in the present results were $100 \%$ susceptible to amoxicillin/ clabvulanic acid, which is in concordance with other studies that BRO beta-lactamases were inhibited by clavulanic acid and its combination with amoxicillin demonstrates $100 \%$ activity [15-17].

The resistance of $M$. catarrhalis to other antimicrobials still has very small significance for the medical practice in Bulgaria. Resistant isolates to quinolones were not detected among tested strains moraxellae. Nevertheless, some isolates with decreased susceptibility to the other groups of antibiotics have been found in the present work. This is in concordance with other reports [16-19]. The found resistance to tetracycline (resp. doxycycline) and sulfamethoxazole+trimethoprim in the examined isolates $M$. catarrhalis was very low and had no in- crease during the study period. Most probably those antimicrobials were not used very often in Bulgaria. In contrast to our work, Pakistan authors reported sulfamethoxazole+trimethoprim resistance rate near 60\% [18]. Our finding of some difference between MICs of azithromycin and clarithromycin were reported by other authors $[8,20]$. The exposure of $M$. catarrhalis to clarithromycin increased the expression of particular components of the efflux pump. Novel mechanisms responsible for resistance to macrolides and quinolones in $M$. catarrhalis were reported and increase of the resistance to these groups antimicrobials in Asia, and Europe were reported [18-21].

\section{CONCLUSIONS}

In summary, the results of examined 20 years period showed that nearly all Bulgarian isolates of $M$. catarrhalis in the last years become resistant to penicillins and first generation cephalosporins via the production of betalactamases, encoded predominantly by bro- 1 gene. The increased resistance to members of second and third generation cephalosporines (cefixime) and slower to other antimicrobials (tetracycline and sulfamethoxazole/trimethoprim) was found. Amoxicillin/clavulanic acid was the most effective antimicrobial. The extended spectrum of beta-lactamases appear to have a significant presence among Bulgarian isolates M. catarrhalis and can compromise the empiric choice of treatment of infections due to this bacterium. The rapid development of resistance in recent years to the most popular antimicrobial agents affects the recommendations regarding the initial approach to therapy.

\section{REFERENCES:}

1. Gergova RT, Petrova G, Gergov S, Minchev P, Mitov I, Strateva T. Microbiological features of upper respiratory tract infections in bulgarian children for the period 1998-2014. Balkan Med J. 2016; 33(6):675-80. [Crossref]

2. Murphy TF. Moraxella catarrhalis, Kingella, and other GramNegative Cocci. In: Mandell, Douglas, and Bennett's Principles and Practice of Infectious Diseases. Authors: Bennett JE, Dolin R, Blaser MJ. 9th Edition. Elsevier. September 12, 2019. Chapter 213. pp.2628-2635e3. [Internet]

3. Pichichero ME. Ten-Year Study of Acute Otitis Media in Rochester, NY. Pediatr Infect Dis J 2016; 35:1027.

4. Georgieva B, Gergova R, Perenovska P, Miteva D, Parina S, Korsun N, et al. Infectious Pathogens that Could Trigger Cough in Children
Outside Winter Epidemic Season. Ann Microbiol Infect Dis. 2019, 2(1), PP 52-57.

5. Gergova R, Markovska R, Mitov I. Antimicrobial resistance and production of beta-lactamases in Bulgarian clinical isolates Moraxella catarrhalis. Ann Microbiol. 2009 Mar; 59:169-172. [Crossref]

6. Micoli F, Costantino P, Adamo R. Potential targets for next generation antimicrobial glycoconjugate vaccines. FEMS Microbiol Rev. 2018 May 1;42(3):388-423. [PubMed] [Crossref]

7. Perez AC, Murphy TF. A Moraxella Catarrhalis Vaccine to Protect Against Otitis Media and Exacerbations of COPD: An Update on Current Progress and Challenges. Hum Vaccin Immunother. 2017 Oct 3; 13(10):2322-2331. [PubMed] [Crossref]
8. Yamada K, Arai K, Saito R. Antimicrobial susceptibility to â-lactam antibiotics and production of BRO âlactamase in clinical isolates of Moraxella catarrhalis from a Japanese hospital. J Microbiol Immunol Infect 2017; 50:386.

9. Lafontaine ER, Wall D, Vanlerberg SL, Donabedian $\mathrm{H}$, Sledjeski DD. Moraxella Catarrhalis Coaggregates With Streptococcus Pyogenes and Modulates Interactions of S. Pyogenes With Human Epithelial Cells. Infect Immun. 2004 Nov; 72(11):6689-93. [PubMed] [Crossref].

10. Perez AC, Pang B, King LB, Tan L, Murrah KA, Reimche JL, et al. Residence of Streptococcus Pneumoniae and Moraxella Catarrhalis Within Polymicrobial Biofilm Promotes Antibiotic Resistance and Bacterial Persistence in Vivo. Pathog Dis. 2014 Apr; 70(3):280-8. [PubMed] [Crossref] 
11. Tan A, Li W-S, Verderosa AD, Blakeway LV, Mubaiwa TD, Totsika M, et al. Moraxella Catarrhalis NucM Is an Entry Nuclease Involved in Extracellular DNA and RNA Degradation, Cell Competence and Biofilm Scaffolding. Sci Rep. 2019 Feb 22; 9(1):2579. [PubMed] [Crossref]

12. Gergova R. Microbiological, Immunological and Molecular Genetic Studies on Moraxella catarrhalis isolates, from patients with respiratory infections [dissertation], [Sofia], Medical University of Sofia, 2008, 211p.

13. European Committee on Antimicrobial Susceptibility Testing. Clinical Breakpoint Tables - bacteria; Version 9.0, 2019.

14. Verhaegh SJC, Streefland A, Dewnarain JK, Farrell DJ, Van Belkum Alex, Hays JP. Age-related genotypic and phenotypic differences in Moraxella catarrhalis isolates from children and adults presenting with respiratory disease in 2001-2002. Microbiol. 2008;154:1178-1184. [PubMed]

15. Khan MA, Northwood JB, Levy
F, Verhaegh SJ, Farrell DJ, Van Belkum A, et al. Bro\{beta\}-lactamase and antibiotic resistances in a global crosssectional study of Moraxella catarrhalis from children and adults. J Antimicrob Chemother. 2010;65:9197. [PubMed]

16. Saito R, Nonaka S, Fujinami Y, Matsuoka S, Nakajima S, Nishiyama $\mathrm{H}$, et al. The frequency of BRO blactamase and its relationship to antimicrobial susceptibility and serum resistance in Moraxella catarrhalis. $J$ Infect Chemother. 2014; 20:6e8 [PubMed]

17. Yanagihara K, Kadota J, Aoki N, Matsumoto T, Yoshida M, Yagisawa $\mathrm{M}$, et al. Nationwide surveillance of bacterial respiratory pathogens conducted by the surveillance committee of Japanese Society of Chemotherapy, the Japanese Association for Infectious Diseases, and the Japanese Society for Clinical Microbiology in 2010: general view of the pathogens' antibacterial susceptibility. J Infect Chemother. 2015;21:410e20. [PubMed]
18. Shaikh SB, Ahmed Z, Arsalan SA, Shafiq S. Prevalence and resistance pattern of Moraxella catarrhalis in community-acquired lower respiratory tract infections. Infect Drug Resist. 2015;8:263-267. [PubMed]

19. Krol-Turminska K, Olender A. Alternations in DNA Gyrase Genes in Low-Level Fluoroquinolone-Resistant Moraxella catarrhalis Strains Isolated in Poland. Infect Drug Resist. 2018 Aug 6;11:1047-1053. [PubMed] [Crossref]

20. Spaniol V, Bernhard S, Aebi C. Moraxella Catarrhalis AcrAB-OprM Efflux Pump Contributes to Antimicrobial Resistance and Is Enhanced During Cold Shock Response. Antimicrob Agents Chemother. 2015 Apr; 59(4):1886-94. [PubMed] [Crossref]

21. Kasai A, Ohta A, Maeda Y, Yamada K, Tao K, Saito R. Novel mechanism responsible for high-level macrolide resistance in Moraxella catarrhalis. Infect Drug Resist. 2018 November, 11, 2137-2140. [PubMed]

Please cite this article as: Gergova R, Markovska R. Antimicrobial resistance of Bulgarian isolates Moraxella catarrhalis during the period 1999-2018. J of IMAB. 2020 Apr-Jun;26(2):3208-3212.

DOI: https://doi.org/10.5272/jimab.2020262.3208

Received: 25/07/2019; Published online: 25/06/2020

\author{
Address for correspondence: \\ Assoc. Prof. Raina Gergova, MD, PhD \\ Department of Medical Microbiology, Faculty of Medicine, Medical Univer- \\ sity of Sofia, Bulgaria, \\ 2, Zdrave str., 1431 Sofia, Bulgaria. \\ E-mail: rtgergova@gmail.com
}

